Ann. Biol. anim. Bioch. Biophys., I968, 8 (4), 537-548.

\title{
ÉTUDE DE L'UTILISATION DIGESTIVE DES ACIDES GRAS CHEZ LE PORC
}

\author{
J. FLANZY, A. RÉRAT, A.-C. FRANÇOIS \\ avec la collaboration technique de Marie-José Lecourtier \\ Station centrale de Nutrition, \\ Centre national de Recherches zootechniques 78 - Joury-en-Josas \\ Institut national de la Recherche agronomique
}

SOMMAIRE

La digestibilité des acides gras ingérés sous forme de triglycérides mixtes a été étudiée chez le Porc. Les CUD moyens apparents obtenus sont les suivants : acide laurique, 90 p. roo ; acide myristique, 67 p. 100 ; acide palmitique, 46 p. I00; acide stéarique, 40 p. I00; acide oléqque, 9o p. 100 ; acide linoléique, 95 p. 100.

La faible digestibilité de certaines graisses est liée à une augmentation de la proportion de composés insolubles retrouvée dans les fèces. Ces composés insolubles, vraisemblablement des sels de $\mathrm{Ca}^{++}$, se formeraient au cours de la digestion, dans la lumière intestinale, surtout à partir des acides saturés, palmitique et stéarique.

L'influence de la structure glycéridique a été étudiée : en effet, quand l'acide palmitique est en position $\beta$ dans la molécule de triglycéride alimentaire (cas du saindoux), il est absorbé sous forme de $\beta$-monoglycéride. Son CUD apparent est alors de $86 \mathrm{p}$. гоo.

\section{INT'RODUCTION}

Dans une revue bibliographique qui analyse les différents facteurs pouvant influer sur la digestibilité des acides gras, THIEuL,IN (I968) montre que, malgré la diversité des expériences et des méthodes utilisées, on peut trouver chez les différents auteurs des conclusions communes. En effet, d'une manière générale, la digestibilité des acides gras saturés décroît avec la longueur de la chaîne, les acides insaturés sont plus digestibles que les acides saturés possédant le même nombre d'atomes de carbone, les acides gras engagés dans les glycérides sont généralement plus digestibles que les acides gras libres. Notre intention est d'appeler l'attention sur deux facteurs de la digestibilité qui ont été souvent ignorés ou négligés. 
Le premier de ces facteurs est la formation, dans la lumière intestinale, de composés insolubles et la présence de ceux-ci dans les fèces ; le second est la structure des glycérides ingérés.

Quelques auteurs ont bien souligné l'importance des formes insolubles d'acides gras dans le phénomène de la digestion des graisses AUGUR, Roliman et DEUEL, (I947) ; AylWARd et WoOd (I962); CARRoli et RICHARDS (I958); CheNG et al. (I949); Crockett et Deuel Jr (I947) ; Deuei, Jr et al., (I949) ; Drenick (I96I) ; Grvens (I9I7) HoLT et al. (I935), Sammons et WIGGs (I960), Yacowitz et al. (I967), mais certains résultats méritent d'être discutés, soit que les expériences aient été effectuées dans des cas pathologiques (stéatorrhées chez l'Homme), soit que les méthodes utilisées n'aient pas été adaptées au dosage de savons et de formes complexes d'acides gras, soit enfin que la matière grasse utilisée dans ces expériences (triglycérides homogènes par exemple) soit trop différente des matières grasses naturelles, par la composition en acides gras et par la structure glycéridique.

Le deuxième facteur est la structure glycéridique. Les graisses naturelles sont composées en grande partie, on le sait, de glycérides mixtes ; autrement dit, les trois hydroxyles de chaque radical glycérol ne sont pas estérifiés par le même type d'acides gras. Les analyses montrent que, dans ces graisses, les acides les moins saturés tendent généralement à occuper les positions " internes " et les acides les plus saturés les positions " externes ". Les seules exceptions actuellement connues sont les graisses de dépôt du Porc et du Pécari, où les chaînes internes sont en grande majorité de l'acide palmitique (SAVARY et al., I957; MATTSON et LuTTON, I958). Quant aux triglycérides " homogènes ", il semble bien qu'ils peuvent exister en proportions importantes seulement dans les corps dont la composition en chaînes est particulièrement simple, un seul acide gras y représentant pius des deux tiers des chaînes (SAVARY et DESNUEILE, Ig6r).

Nous nous sommes proposé, dans un travail préliminaire sur Porc, de tenir compte de ces différents facteurs. Cela nous permettra, dans un deuxième temps, de généraliser notre méthode de travail et de rechercher s'il existe des lois applicables aux différentes espèces domestiques, Porc, Veau, Poulet.

Pour ces recherches, nous avons mis au point une méthode d'analyse et d'isolement des fractions lipidiques fécales, qui permet de caractériser et de doser les acides gras sous leurs différentes formes (TOUL,LEC et al., Ig68).

\section{MÉTHODES}

L'expérience consıste à donner à des porcs soit un régime lipidoprive, soit des régimes contenarit une proportion déterminée d'un corps gras dont la structure et la composition en chaînes sont con: nues. Comme corps gras, nous avons pris soit du saindoux, soit divers mélanges de graisses que nous appellerons $\mathrm{X}_{1}, \mathrm{X}_{2}$ et $\mathrm{X}_{3}$. Les essais ont porté sur deux porcs placés dans des cages à métabolisme dès le poids de $50 \mathrm{~kg}$. Ces animaux ont d'abord été soumis à un régime lipidoprive pendant ro jours. Puis, ils ont été soumis à un régime croisé, chacun des deux animaux recevant alternativement pendant une période de ${ }_{5} 5$ jours l'aliment $X_{1}$ ou l'aliment $X_{2}$. Entre chacun des régimes expérimentaux, les animaux recevaient un régime lipidoprive pendant une semaine.

L'aliment $\mathrm{X}_{3}$ et l'aliment contenant du saindoux ont été substitués aux aliments $\mathrm{X}_{1}$ et $\mathrm{X}_{2}$ au cours d'une période de $\mathrm{r} 5$ jours. La durée de l'expérience a été de 3 mois. 
Le tableau I donne la composition pondérale des régimes.

Dans le tableau 2, on trouve la composition en chaînes des graisses $X_{1}, X_{2}$ et $X_{3}$. Celles-ci ont été obtenues en mélangeant des huiles de coprah, de tournesol et de coton avec du beurre de cacao (ce dernier apportant les chaînes stéariques). On notera que, dans ces divers mélanges, les proportions de chaînes palmitiques sont très voisines ; il en est d'ailleurs de même pour les teneurs en acide stéarique. Les autres constituants peuvent quelque peu varier, mais leurs proportions correspondent aux cas que l'on peut rencontrer dans la pratique. Ce même tableau 2 donne également l'analyse des acides gras du saindoux que nous avons utilisé pour voir l'influence de la structure glycéridique sur le coefficient d'utilisation digestive des chaînes palmitiques. En effet, dans les mélanges $\mathrm{X}_{1}, \mathrm{X}_{2}$ et $\mathrm{X}_{3}$, les chaînes palmitiques et stéariques sont essentiellement fixées sur les hydroxyles primaires du glycérol, tandis que, pour le saindoux, si les chaînes stéariques sont encore en "position externe *, la presque totalité des chaînes palmitiques se trouve sur les hydroxyles secondaires, c'est-à-dire en "position interne".

TABLEAU I

Composition des régimes (p. Ioo en poids)

\begin{tabular}{|c|c|c|c|c|c|}
\hline & Lipidoprive & $X_{1}$ & $\mathrm{X}_{2}$ & $\mathbf{X}_{3}$ & Saindoux \\
\hline Lait écrémé Spray . . . . . . . . . & 30 & 35 & 35 & 35 & 35 \\
\hline Amidon...$\ldots \ldots \ldots \ldots \ldots$ & 58,8 & 39 & 39 & 39 & 39 \\
\hline$\left(x_{1} \ldots \ldots\right.$ & - & 13 & & & \\
\hline Mélange graisses $\left\{\begin{array}{l}X_{2} \ldots \ldots \\
X^{2} \ldots\end{array}\right.$ & - & & 13 & 13 & \\
\hline Saindoux $\ldots \ldots \ldots \ldots \ldots \ldots \ldots$ & - & & & & 13 \\
\hline Cellulose $\ldots \ldots \ldots \ldots \ldots \ldots$ & 4,3 & 5 & 5 & 5 & 5 \\
\hline Mélange minéral $\ldots \ldots \ldots \ldots \ldots$ & 5,2 & 6 & 6 & 6 & 6 \\
\hline Mélange vitaminique $\quad \ldots \ldots \ldots$ & 1,7 & 2 & 2 & 2 & 2 \\
\hline
\end{tabular}

TABLEAU 2

Composition en acides gras des graisses du régime (esters méthyliques, p. roo en poids du total)

\begin{tabular}{|c|c|c|c|c|c|c|}
\hline \multirow{2}{*}{ Régimes } & \multicolumn{6}{|c|}{ Acides gras } \\
\hline & $\mathrm{C}_{12}$ & $\mathrm{C}_{\mathbf{1 4}}$ & $\mathrm{C}_{16}$ & $\mathrm{C}_{\mathbf{1 8}}$ & $\mathrm{C}_{18: 1}$ & $\mathrm{C}_{18: 2}$ \\
\hline $\begin{array}{l}\mathbf{x}_{1} \ldots \ldots \ldots \ldots \ldots \ldots \\
\mathbf{x}_{2} \ldots \ldots \ldots \ldots \ldots \ldots \ldots \\
\mathbf{x}_{3} \ldots \ldots \ldots \ldots \ldots \ldots \\
\text { Saindoux } \ldots \ldots \ldots \ldots\end{array}$ & $\begin{array}{r}8,4 \\
12,1 \\
15,3\end{array}$ & $\begin{array}{l}3,6 \\
5,6 \\
6,3\end{array}$ & $\begin{array}{l}19,7 \\
19,2 \\
19,6 \\
26,8\end{array}$ & $\begin{array}{l}21,6 \\
22,4 \\
19,9 \\
15,1\end{array}$ & $\begin{array}{l}36,4 \\
27,4 \\
28,1 \\
50,3\end{array}$ & $\begin{array}{r}10,3 \\
13,3 \\
10,7 \\
7,8\end{array}$ \\
\hline
\end{tabular}

Le régime lipidoprive contenait 0, I I p. Ioo de matière grasse (par rapport à la matière sèche).

La consommation des animaux était égalisée en énergie et en azote.

Les fèces ont été prélevées chaque jour, stockées pour être rassemblées à l'issue d'une même période, homogénéisées et séchées à $60^{\circ} \mathrm{C}$ dans une étuve à circulation. Nous avions vérifié que la lyophilisation des fèces n'était pas indispensable pour éviter l'altération des graisses (autoxydation et transformations microbiennes). Les résultats de l'analyse des matières grasses étaient identiques, quel que soit le traitement.

L'analyse des matières grasses des fèces et du régime lipidoprive a été effectuée comme nous l'indiquons en détail dans une autre publication (Toullec et al., 1968). Nous ne citerons ici que le principe de la méthode. Elle comprend trois étapes : 
$\mathrm{I}^{\circ}$ les lipides apolaires sont extraits par la méthode de FoLCH (I957) et constituent ce que nous appelons " extrait Folch" (E.F.) ;

$2^{\circ}$ la phase aqueuse de lavage de l'extrait est acidifiée et les acides gras extraits par l'éther de pétrole constituent la fraction qui est appelée "savons solubles";

$3^{\circ}$ le résidu de la première extraction Folch est acidifié, afin de libérer et d'extraire les acides gras précipités ou combinés dans le produit initial. Les acides gras ainsi obtenus constituent la fraction qui est appelée " savons insolubles "(S.I.). D'une manière générale, dans le cas des fèces, nous avons constaté que les " savons solubles " représentaient une fraction négligeable de l'extrait. Nous l'avons donc arbitrairement ajoutée à l'extrait Folch, pour la simplification de nos calculs.

Les acides gras, libérés par saponification des différentes fractions, ont été ensuite identifiés et dosés par chromatographie en phase gazeuse. Les conditions expérimentales étaient les suivantes : la longueur de la colonne était de $3 \mathrm{~m}$ et son diamètre de $4 \mathrm{~mm}$ environ. Le support, du chromosorb WHMDS, était imprégné de I 2 p. roo de succinate de diéthylène glycol. La température de la colonne était de ${ }^{8} 80^{\circ} \mathrm{C}$. Le calcul de la surface des pics était réalisé par un intégrateur DISC. Dans nos conditions expérimentales, le nombre des plateaux théoriques de la colonne était de 2500 . Dans l'expérience avec du saindoux, nous n'avons dosé que les acides palmitique, stéarique, oléique et linoléique. Dans les autres cas, nous avons dosé en plus les acides laurique et myristique. Les résultats sont exprimés en p. 100 des esters méthyliques dosés.

\section{RÉSULTATS}

\section{Matières grasses "endogènes"}

Nous avons déterminé la matière grasse excrétée quand 1'animal était soumis au régime lipidoprive. Ce régime contenait toutefois 0, I I p. Ioo (de la M. S.) de matière grasse. Nous avons ainsi calculé que l'animal soumis à un régime lipidoprive, excrétait par jour 3 à $4 \mathrm{~g}$ de lipides, alors que l'aliment ingéré en contenait de I à $2 \mathrm{~g}$. A titre de comparaison, en régime lipidique, le même animal excrétait environ, en moyenne, 50 à $60 \mathrm{~g}$ de graisses. La composition en acides gras des fèces en régime lipidoprive est donnée dans le tableau 3 .

\section{TABLEAU 3}

Composition en acides gras des fèces en régime lipidoprive (esters méthyliques, p. 100 en poids du total)

\begin{tabular}{c|c|c|c|c|c}
\hline \hline $\mathrm{C}_{12}$ & $\mathrm{C}_{14}$ & $\mathrm{C}_{18}$ & $\mathrm{C}_{18}$ & $\mathrm{C}_{18: 1}$ & $\mathrm{C}_{18: 2}$ \\
\hline 2,4 & 4,6 & 34,3 & 41,1 & 14,5 & 3,1 \\
\hline
\end{tabular}

BAYLEY et LEWIS (I965 a) avaient déjà remarqué que les acides gras d'origine endogène, qu'ils proviennent de l'hydrogénation des acides gras alimentaires ou qu'ils proviennent des desquamations intestinales, contenaient une forte proportion d'acides saturés.

\section{Digestibilité globàle apparente des différentes graisses}

Nous n'avons pas tenu compte, dans nos calculs, des matières grasses endogènes, que nous aurions pu assimiler aux matières grasses excrétées en régime lipidoprive, comme le font BAYLEY et LEWIS ( I965 b). Nous avons préféré calculer la digestibilité apparente : en effet Scribante et FAVARGER (I954), BUENSOD et FAVARGER (I956), 
Planche et al. (Ig66), WeRNER (I965) ont montré que l'excrétion des lipides endogènes était influencée par la nature et la quantité des graisses du régime. $\mathrm{Si}$, donc, nos résultats donnent un ordre de grandeur sur la quantité, la nature et la composition de ces lipides endogènes en régime lipidoprive, rien ne nous permet d'introduire des valeurs pour un calcul de la digestibilité réelle en régime lipidique.

La digestibilité apparente moyenne de chacune des graisses étudiées est donnée dans le tableau 4.

TABLEAU 4

Digestibilité apparente globale moyenne des graisses étudiées

\begin{tabular}{|c|c|}
\hline Régimes & $\begin{array}{l}\text { Coefficient de digestibilité apparente } \\
\text { (p. 100) }\end{array}$ \\
\hline 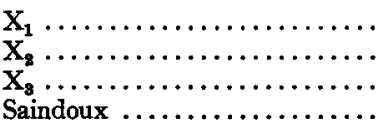 & $\begin{array}{l}69,0 \\
70,0 \\
72,3 \\
84,4\end{array}$ \\
\hline
\end{tabular}

Il ressort de ce tableau que la digestibilité du saindoux est la plus élevée, celle des trois autres graisses se situant à $70 \mathrm{p}$. Ioo environ.

\section{Digestibilité apparente des acides gras}

Nous avons calculé les valeurs correspondant au coefficient de digestibilité apparente moyen établi sur trois périodes de $\mathrm{I}_{5}$ jours pour l'aliment $\mathrm{X}_{2}$ et sur deux périodes de $I 5$ jours pour l'aliment $X_{1}$. Les valeurs correspondant au coefficient de digestibilité apparente de l'aliment $X_{3}$ et du saindoux ont été établies sur une période de I5 jours. Les résultats sont indiqués dans le tableau 5.

TABLEAU 5

Coefficients de digestibilité apparente des acides gras suivant le régime (en p. 100)

\begin{tabular}{|c|c|c|c|c|c|c|}
\hline \multirow{2}{*}{ Régimes } & \multicolumn{6}{|c|}{ Acides gras } \\
\hline & $\mathrm{C}_{12}$ & $\mathrm{C}_{14}$ & $\mathrm{C}_{16}$ & $\mathrm{C}_{18}$ & $C_{18: 1}$ & $C_{18: 2}$ \\
\hline $\begin{array}{l}\mathbf{x}_{1} \ldots \ldots \ldots \ldots \ldots \ldots \\
\mathbf{x}_{8} \ldots \ldots \ldots \ldots \ldots \ldots \ldots \\
\mathbf{x}_{8} \ldots \ldots \ldots \ldots \ldots \ldots \\
\text { Saindoux } \ldots \ldots \ldots \ldots\end{array}$ & $\begin{array}{l}87,8 \\
88,0 \\
90,5\end{array}$ & $\begin{array}{l}65,3 \\
67,3 \\
68,7\end{array}$ & $\begin{array}{l}45,5 \\
46,4 \\
49,1 \\
86,2\end{array}$ & $\begin{array}{l}36,0 \\
42,6 \\
41,9 \\
53,1\end{array}$ & $\begin{array}{l}89,5 \\
90,8 \\
92,4 \\
90,9\end{array}$ & $\begin{array}{l}94,7 \\
94.8 \\
95,3 \\
97,0\end{array}$ \\
\hline
\end{tabular}

Nos résultats confirment les données généralement admises sur la digestibilité des acides gras : celle-ci est élevée pour les acides insaturés et les acides saturés jusqu'au $\mathrm{C}_{12}$ et elle décroît de $90 \mathrm{p}$. Ioo à $40 \mathrm{p}$. Ioo pour les acides saturés, en fonction de la longueur de chaîne, de $\mathrm{C}_{12}$ à $\mathrm{C}_{18}$. 
Nous avons calculé par ailleurs, pour chacun des deux porcs, la digestibilité des acides gras entrant dans les régimes $\mathrm{X}_{1}$ et $\mathrm{X}_{2}$ (tabl. 6).

Les valeurs obtenues sont presque identiques dans l'un et l'autre cas et sont donc indépendantes de l'animal.

TABLEAU 6

Coefficients de digestibilité apparente des acides gras suivant l'animal (en p. 100)

\begin{tabular}{|c|c|c|c|c|c|c|}
\hline & \multicolumn{6}{|c|}{ Acides gras } \\
\hline & $\mathrm{C}_{12}$ & $\mathrm{C}_{14}$ & $\mathrm{C}_{16}$ & $\mathrm{C}_{18}$ & $\mathrm{C}_{18: 1}$ & $\mathrm{C}_{18: 2}$ \\
\hline $\begin{array}{l}\text { Porc } 1 \\
\text { Porc } 2\end{array}$ & $\begin{array}{l}87,5 \\
88,4\end{array}$ & $\begin{array}{l}66,0 \\
67,1\end{array}$ & $\begin{array}{l}45,8 \\
46,3\end{array}$ & $\begin{array}{l}39,8 \\
40,5\end{array}$ & $\begin{array}{l}90,4 \\
90,1\end{array}$ & $\begin{array}{l}95,0 \\
94,4\end{array}$ \\
\hline
\end{tabular}

4. Composition en acides gras

des différentes fractions lipidiques excrétées dans les fèces

Pour chacune des graisses étudiées, nous avons mesuré dans les fèces la part respective de l'extrait Folch (additionné de la fraction "savons solubles ") et de la fraction "savons insolubles", ainsi que la composition de chacune d'elles en acides gras. Les résultats sont consignés dans le tableau 7 .

\section{TABLEAU 7}

Composition en acides gras des fractions

"Extrait Folch" (E.F.) et "Savons insolubles" (S.I.)

\begin{tabular}{|c|c|c|c|c|c|c|c|}
\hline \multirow{2}{*}{ Régimes } & \multirow{2}{*}{$\begin{array}{l}\text { P. } 100 \text { respectifs } \\
\text { de E. F. et S. I. }\end{array}$} & \multicolumn{6}{|c|}{ Composition en acides gras (p. 100 du total) } \\
\hline & & $\mathrm{C}_{12}$ & $\mathrm{C}_{14}$ & $\mathrm{C}_{16}$ & $\mathrm{C}_{18}$ & $\mathrm{C}_{18: 1}$ & $\mathrm{C}_{18: 2}$ \\
\hline \multirow{2}{*}{$\mathrm{X}_{1}$} & E. F. 20,9 & 5,5 & 4,8 & 30,5 & 30,8 & 24,3 & 3,9 \\
\hline & S. I. 79,1 & 2,7 & 3,7 & 35,6 & 48,5 & 8,3 & 1,0 \\
\hline \multirow{2}{*}{$\mathrm{x}_{2}$} & E. F. 21,0 & 8,8 & 7,9 & 30,1 & 29,4 & 18,4 & 5,9 \\
\hline & S. I. 79,0 & 4,0 & 5,4 & 36,3 & 47,4 & 5,6 & 1,2 \\
\hline \multirow{2}{*}{$\mathbf{X}_{\mathbf{3}}$} & E. F. 18,2 & 8,8 & 8,7 & 29,0 & 26,2 & 21,3 & 6,0 \\
\hline & S. I. 81,8 & 4,5 & 6,9 & 37,7 & 45,1 & 4,9 & 0,9 \\
\hline \multirow{2}{*}{ Saindoux } & E. F. 26,9 & & & 18,0 & 24,4 & 53,8 & 3,8 \\
\hline & S. I. 73,1 & & & 25,8 & 53,0 & 20,5 & 0,7 \\
\hline
\end{tabular}

Nous constatons que, dans tous les cas, la fraction insoluble est plus riche en acides saturés longs (palmitique et stéarique) que la fraction " extrait Folch ».

\section{DISCUSSION E'T CONCLUSION}

BAYLEY et LEWIS ( $1965 b$ ) ont tenté de mesurer la digestibilité " vraie " des acides gras chez le Porc, en utilisant comme source de matières grasses des acides gras libres, des triglycérides homogènes, des graisses commerciales. Les valeurs obtenues par ces 
auteurs sont très proches des nôtres pour les acides insaturés, légèrement supérieures et plus variables pour les acides saturés. Nous avons dit pourquoi le calcul du CUD vrai nous paraissait erroné, si on l'établissait à partir de quantités d'acides gras excrétés en régime lipidoprive. On ne peut parfaitement connaître ce coefficient qu'en utilisant des graisses marquées sur tous les acides gras (ScRIBANTE et Favarger, I954; WERNER, I965).

La digestibilité globale moyenne des graisses étudiées est plus faible que celle admise généralement, sauf dans le cas du saindoux. Ceci résulte du fait que notre méthode de dosage extrait des fèces la totalité des acides gras, sous quelque forme qu'ils soient.

La fraction " insoluble " représente, dans tous les cas, une fraction importante des lipides fécaux (80 p. xoo environ), fraction qui n'entre pas dans le calcul de la digestibilité, lorsque les lipides sont déterminés par les méthodes classiques d'extraction aux solvants. Comme 1'ont fait quelques auteurs avant nous (AUGUR et al., I947; CARroli, I958 ; Clement et Clement, I958; Williams, I962; Fleischman et al., I966; Yacowitz et al., I967), il est donc indispensable de ne doser les lipides fécaux qu'après une hydrolyse acide, pour libérer les acides gras des composés insolubles.

La nature de ces composés insolubles n'est pas, toutefois, clairement définie. Pour de nombreux auteurs, Carroli (r958), CARroli et Richards (I958), Dranick, (I96I), LuTWAK et al., (I964), WERNER et LuTWAK (I963), YACOWITZ et al., (I967), ces composés seraient constitués de savons insolubles de $\mathrm{Ca}^{++}$, qui seraient différents des complexes phosphorés de cafium et d'acide oléique, isolés dans des fèces par SwELL et al. (I956) et, après eux, CARRoli, et RICHARDs (I958), RICHARDS et CARROLL (I959). Fleischman et al. (I966), Petrierson (I964), Yacowitz et al. (I967) établissent une relation directe entre l'augmentation de la teneur en calcium de la ration et l'augmentation de l'excrétion $\mathrm{du} \mathrm{Ca}^{++}$et des acides gras. Comment ces composés se formentils? Pourquoi seraient-ils formés surtout à partir des acides gras saturés ? La présence de ces formes insolubles et celle d'une solution micellaire sont-elles compatibles dans la lumière intestinale, dans l'hypothèse, évidemment, où la mise en solution micellaire est une étape nécessaire de la digestion et correspond bien à la réalité physiologique (HOFMANN et Borgstrom, Ig62; FrEEMAN et al., I967)?

Pour répondre en partie à ces questions, il faut rappeler certaines conclusions des travaux de Hofmann ( $1963 a$ ). Cet auteur a montré notamment que l'addition de $\mathrm{Ca}^{++}$aux solutions micellaires d'acides gras et de sels biliaires avait pour conséquence

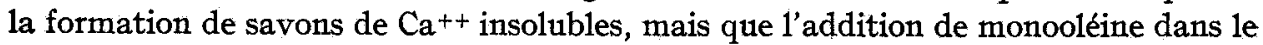
milieu réactionnel inhibait la formation de ces savons. Dans nos régimes, l'acide oléique constitue $25 \mathrm{p}$. Ioo au minimum des acides gras alimentaires et la monooléine provenant de la lipolyse de ces graisses devrait donc inhiber la formation des sels de $\mathrm{Ca}^{++}$ insolubles. Or, il n'en est rien. Il faut supposer que, pour un acide gras, la formation du composé insoluble se fait soit avant cette mise en solution micellaire, soit après celle-ci.

Dans le premier cas, la lipase pancréatique libérerait à partir des triglycérides émulsionnés les acides gras qui précipiteraient immédiatement sous forme insoluble ; il faudrait donc supposer que les micelles déjà formées sont "saturées " et ne peuvent plus " redissoudre" les formes insolubles. Puisque les acides saturés sont en général en position $\alpha$, donc libérés les premiers, et que les monoglycérides sont surtout constitués de monooléine, on pourrait conclure à une compétition entre les acides saturés 
et la monooléine: Savary et Constantin ( 1967 ), ainsi que Hofmann et BorgsTROM (1962) sont arrivés à des conclusions analogues in vitro en étudiant la solubilisation des acides saturés dans des micelles contenant de l'acide oléique ou de la monooléine.

Dans le deuxième cas, nos résultats confirmeraient l'hypothèse suivant laquelle les micelles mixtes de sels biliaires et d'acides gras seraient détruites avant l'absorption proprement dite, soit dans le film de mucus qui tapisse les cellules épithéliales absorbantes, soit au contact de ces mêmes cellules (VODOVAR et FLANZY, I966; IsSELBACHER, I967). Dans ces conditions, les acides gras retrouveraient leurs propriétés chimiques et notamment leur aptitude à précipiter avec les ions $\mathrm{Ca}^{++}$. En effet, bien que les acides gras ne soient pas totalement ionisés au $\mathrm{pH}$ de l'intestin à ce niveau (Borgstrom, I967; Hofmann et Borgstrom, I963), l'équilibre de dissociation est entièrement déplacé vers la formation du composé insoluble.

Nous avons vu que la structure des glycérides ingérés pouvait expliquer que les formes insolubles soient surtout constituées in vivo d'acides gras saturés. D'ailleurs, il en est de même " in vitro " quand on fabrique des sels de $\mathrm{Ca}^{++}$à partir des acides gras libres. CLEMENT et CLEMENT (I958) ont montré que l'indice d'iode des savons formés à partir d'un mélange d'acide oléique et d'acides saturés est nettement inférieur à celui du mélange d'acides gras primitif. Plus récemment, nous avons montré (TouLLEC et al., I968) que les savons de calcium obtenus par addition d'une solution de $\mathrm{Cl}_{2} \mathrm{Ca}$ à une solution alcoolique équimoléculaire des acides palmitique, stéarique, oléique, linoléique, en quantité telle qu'il y ait un quant d'atome-gramme de $\mathrm{Ca}^{++}$ pour chaque molécule-gramme d'acides gras, sont composés pour $97,5 \mathrm{p}$. roo d'acides saturés, soit 58,7 p. Ioo d'acide stéarique et 38,8 p. Ioo d'acide palmitique.

En conclusion, on peut dire que les acides saturés longs font diminuer la digestibilité globale d'une matière grasse, parce $q u^{\prime}$ ils forment avec le $\mathrm{Ca}^{++}$des composés insolubles qui ne sont pas absorbés par la paroi intestinale. Cette réaction est plus rapide quand les acides gras sont ingérés sous forme libre que quand ils sont combinés au glycérol. C'est ce qui explique que les acides gras soient moins bien absorbés, sous forme libre que sous forme de glycéride (CoNSTANTIN et SAVARY, I965; HOAGLAND et SNIDER, I944; MaTtil, I946; RENNER et HiLL, I958; WeRNER, I965).

La digestibilité du saindoux est plus élevée que celle des autres graisses. Cette augmentation est due presque uniquement à une meilleure absorption des acides saturés, l'acide stéarique et surtout l'acide palmitique. En effet, prenons comme exemple le lot $\mathrm{X}_{1}$ et le lot " saindoux" : nous constatons que la teneur en acides saturés longs (stéarique et palmitique) est respectivement de 4I,3 p. Ioo et de 4I,9 p. Ioo, donc pratiquement identique dans les deux cas. Or, un calcul effectué à partir des tableaux 2 et 5 montre que la digestibilité de ces deux acides gras, dans le cas du saindoux, fait croître la digestibilité globale de 14,4 p. Ioo, alors que l'augmentation totale de la digestibilité du saindoux est de I5,4 p. IOo. C'est donc uniquement à l'amélioration de la digestibilité des acides saturés que l'on doit l'augmentation de la digestibilité globale.

On pourrait dire que les acides saturés sont mieux absorbés dans le régime saindoux, parce que, dans ce régime, on a remplacé les acides laurique et myristique des régimes $\mathrm{X}_{1}, \mathrm{X}_{2}$ et $\mathrm{X}_{3}$ par les acides oléique et linoléique, ce qui abaisse le point de fusion. B'autre part, on a augmenté le pourcentage d'acide palmitique aux dépens du pourcentage d'acide stéarique, ce qui contribue également à abaisser le point de 
fusion. En effet, certains auteurs, notamment CALL,OWAY et KURTZ (I956), Young et GARRETT. (1963) ont remarqué que les acides saturés étaient moins bien absorbés, quand ils étaient ingérés seuls qu'en présence d'acides insaturés. Parallèlement, la mise en solution micellaire des acides saturés en présence d'acide oléique, que ce dernier soit sous forme libre, ou bien sous forme de monoglycéride, a comme conséquence directe 1'abaissement du point de fusion (Hofmann, I963 $b$; Hofmann et BorgsTrom, I962). Plus récemment, Savary (rg66) a montré que la solubilité des acides saturés (palmitique et stéarique) dans les micelles de sels biliaires est augmentée proportionnellement à la quantité d'acide oléique ajouté, mais que la quantité d'acides saturés solubilisée passe par un maximum (SAVARY et Constan'rin, r967), quand la quantité de chaînes oléiques augmente : le rapport de la quantité des chaînes d'acide oléique à la quantité des chaînes d'acide palmitique est alors de l'ordre de Io. Or, dans nos expériences le rapport des chaînes insaturées aux chaînes saturées varie de 0,4 à 0,58 , c'est-à-dire qu'elles ne peuvent solubiliser environ que 5 chaînes sur Ioo d'acides saturés. De plus, si la teneur en acides insaturés avait augmenté la digestibilité des acides saturés, il aurait fallu que la digestibilité des acides saturés (palmitique et stéarique) dans le lot $X_{1}$, qui contient $46,7 \mathrm{p}$. Ioo d'acides insaturés, soit supérieure à celle du $\operatorname{lot} X_{3}$, qui n'en contient que 38,7 p. Ioo ; or, il n'en est rien, elle est au contraire légèrement supérieure dans le lot $X_{3}$. On ne peut donc pas conclure que, dans nos expériences, les acides insaturés jouent un rôle direct ou indirect dans la digestibilité globale des acides gras.

En revanche, la meilleure digestibilité de l'acide palmitique peut être la conséquence de la structure particulière du saindoux, comme l'avaient déjà signalé RENNER et HILL (I96I). On remarque, en effet, que la digestibilité des chainnes stéariques est la même pour les régimes $X_{1}, X_{2}, X_{3}$ d'une part, et pour le saindoux, d'autre part, tandis que celle des chaînes palmitiques est nettement meilleure dans le second cas. La seule différence que l'on aperçoive entre les deux séries d'expériences, c'est que les chaînes palmitiques ne sont pas localisées de la même façon dans les triglycérides, tandis que les chaînes stéariques, elles, sont toujours en position externe. Dans le saindoux, les chaînes palmitiques se trouvent en position $\beta$ dans les molécules de triglycérides. Or, la lipase pancréatique hydrolysant de préférence les chaînes situées en position $\alpha$, ces acides saturés se trouveraient dans la lumière intestinale sous forme de monoglycérides qui traverseraient ainsi la paroi intestinale. Dans les autres graisses, les acides saturés, qui sont en position externe dans la molécule de triglycéride, sont libérés par la lipase pancréatique sous forme d'acides gras libres, qui peuvent soit cristalliser, soit même donner des composés insolubles, comme nous l'avons vu plus haut. RENNER et HIL, avaient d'ailleurs montré qu'en intérestérifiant les glycérides du saindoux, c'est-a-dire en augmentant le nombre de chaînes saturées en position $\alpha$, la digestibilité du saindoux diminuait chez le Poulet. En revanche, il semblerait que l'influence de la structure glycéridique soit moins nette dans le cas du Rat (SAVARY et al., I96I).

Des conclusions pratiques ressortent de nos résultats concernant l'utilisation digestive des matières grasses. Pour augmenter la digestibilité des graisses, il faut choisir des matières premières qui contiennent le minimum d'acides saturés. Or, ceux-ci sont indispensables pour une bonne utilisation métabolique des matières grasses (Hopkrns et al., I955; FLANZY et $a l$., à paraître). Le rapport optimum acides saturés/acides insaturés étant donc déterminé pour une bonne utilisation métabolique, on 
pourra alors augmenter l'utilisation digestive en interestérifiant, par exemple, les matières grasses, choisies pour leur composition en acides gras. Ce procédé technologique, qui a pour but d'augmenter le nombre de chaînes saturées en position $\beta$, ne doit pas évidemment être appliqué au cas du saindoux. Il ne faut pas non plus faire subir ce traitement à des corps gras comme le cacao et le karité, car l'intérestérification entraîne, dans ce cas, la formation de glycérides trisaturés.

L'autre conclusion pratique est que le composé minéral doit être calculé en fonction du pourcentage et de la nature des matières grasses introduites dans la ration des animaux. Cette étude fait l'objet d'un travail en cours.

Reçu pour publication en juin 1968.

\section{SUMMARY}

DIGESTIVE UTILIZATION OF FATTY ACIDS BY PIGS

Digestibility of fatty acids ingested in the form of mixed triglycerides was studied in pigs. The lipip excrated were separated into two fractions; the first, soluble in the solvents, corresponded to glycerides and free fatty acids ; the second, soluble in the solvents after acidification of the medium, corresponded to the "insoluble soaps". The fatty acid composition of each fraction was estimated by chrometography in the gaseous phase. Average percentage apparent digestibilities obtained were as follows : lauric acid 90 , myristic acid 67 , palmitic acid 46 , stearic acid 40 , oleic acid 90 and linoleic acid 95 .

The low digestibility of certain fats was related to an increase in the proportion of insoluble components recovered in faeces. These insoluble components, probably salts of $\mathrm{Ca}^{++}$, would be formed in the lumen of the intestine in the course of digestion, particularly from the saturated acids, palmitic and stearic. These hypotheses are discussed and considered in relation to the conclusions which derive from the micellar theory of absorption.

The influence of glyceride structure was studied. In fact, when palmitic acid was situated in the $\beta$ position in the triglyceride molecule in the feed (as in lard) it was absorbed as $\beta$-monoglyceride. Its apparent digestibility was then 86 per cent.

Practical conclusions result from this work, both for the choice of fats for feeds and for the technological procedures to be used (interesterification).

\section{RÉFÉRENCES BIBLIOGRAPHIQUES}

Augur V., Rollman H.S., Deuel Jr. H. J., 1947. The effect of crude lecithin on the coefficient of digestibility and the rate of absorption of fat. J. Nutr., $\mathbf{8 3}$, I 77-186.

AYWARD F., WOOD P.D.S., I962. Lipid excretion. 2. Fractionation of human frecal lipids. Brit. J. Nutr. 16, $345-360$.

BAYLEY H. S., LEwIS D, 1965a. The use of fats in pig feeding. I. Pig fæcal fat not of immediate dietary origin. J. agric. Sci., 64, 367-372.

BAYLEY H. S., LEwIS D., $1965 b$. The use of fats in pig feeding. II. The digestibility of various fats and fatty acids. J.agric. Sci, 64, 373-378.

Borgström B., 1967. Partition of lipids between emulsifield oil and micellar phases of glyceride-bile salt dispersions. J. Lipid Res., 8, 598-608.

Buensod M., Favarger P., 1956. Observations sur la digestibilité des acides palmitique et stéarique et de leurs esters glycériques chez le rat. Helv. physiol. pharmac. Acla, 14, 299-303.

Calloway D. H., Kurtz G. W., I956. The absorbility of natural and modified fats. Fd. Res., 21, 621629.

Carroll K. K., I958. Digestibility of individual fatty acids in the rat. J. Nutr., 64, 339-4I0.

Carroll K. K., RIChards J. F., I958. Factors affecting digestibility of fatty acids in the rat. J. Nulr., 64, $41 \mathrm{I}-424$. 
Cheng A. L. S., Morehouse M. G., Deuel Jr. H. J., 1949. The effect of the level of dietary calcium and magnesium on the digestibility of fatty acids, simple triglycerides and some natural and hydrogenated fats. J. Nuir., 87, $237-250$.

Clément J., Clement G., i958. Présence de savons relativement saturés à différentes étapes de ld digestion, de la résorption et du transport des lipides. Oléagineux, 18, 103-105.

Constantin M. J., Savary P., 1965. Sur l'incorporation des chaînes stéariques dans les chylomicrons lymphatiques du Rat. Biochim. biophys. Acta., 106, 248-260.

CrocketT M. E., Deues Jr. H. J., I947. A comparison of the coefficient of digestiblity and the rate of absorption of several natural and artificial fats as influenced by melting point. J. Nutr., 88, 187-194.

Deuel Jr. H. J., Johnson R. M., Cai.bert C. E., Gardner J., Thomas B., I949, Studies on the comparative nutritive value of fats. I2. The digestibility of rapeseed and cottonseed oils in human subjects. J. Nutr., 83, 369-379.

DrEnick E. J., 1961. The influence of ingestion of calcium and other soapforming substances on fæcal fat. Gastroenterology, 41, 242-244.

Flanzy J., Rerat A., FrançoIs A. C., Étude de l'utilisation métabolique des acides gras chez le Porc. Ann. Biol. anim. Bioch. Biophys., (à paraître).

Fleischman A. I., Yacowitz H., Hayton T., Bierenbaum M. L., ig66. Effects of dietary calcium upon lipid metabolism in mature male rats fed beef tallow. $J$. Nutr., 88, 255-260.

Folch J., Lees M., Sloane Stanley G. H., I957. A simple method of the isolation and purification of total lipids from animal tissues. J. biol. Chem., 228, 497-509.

Freeman C. P., Arrison E. F., Noakes D. E., Hill K. J., 1967. The absorption of micellar fat in pigs. Proc. Nutr. Soc., 86, VII.

Givens M. H., I 917 . Studies in calcium and magnesium metabolism. III. The effect of fat and fatty acid derivatives. J. biol. Chem., 31, 44I-444.

HoAgland R., SNIDER G. G., I944. Digestibility of certain higher saturated fatty acids and triglycerides J. Nutr., 26, $219^{-225}$.

Hofmann A. F., $1963 a$. Further studies on the intraluminal phase of fat digestion : factors influencing the behaviour of fatty acids in bile-salt solutions. VI Congrès de Nutrition, Édimbourg, 501.

HoFmanN A. F., 1963 b. The behaviour and solubility of monoglycerides in dilute micellar bile-salt solution Biochim. biophys. Acta, 70, 306-316.

Hofmann A. F., Borgstrom B., I 62 . Physico-chemical state of lipids in intestinal content during their digestion and absorption. Fedn. Proc., 21, 43-50.

HOFMANN A. F., BORGSTRöM B., 1963. Hydrolysis of long-chain monoglycerides in micellar solution by pancreatic lipase. Biochim. biophys. Acta, 70, 3I 7-33I.

Holt L. E., Tidwell H. C., KIrK C. M., Cross D. M., Neale S., I935. Studies in fat metabolism. I. Fat absorption in normal infants. J. Pedial., 6, 427-480.

Hopkins C. Y., Murray T. K., Campbell. J. A., 1955. Optimum ratio of saturated to mono-unsaturated fatty acids in rat diets. Can. J. Bioch. Physiol., 88, I $047^{-1054}$.

ISSELBacher K. J., 1967. Biochemical aspects of lipid malabsorption. Fedn. Proc. 26, 1420-1425.

Lutwak L., Laster L., Gitelman H. J., Fox M., Whedon G. D., 1964. Effects of high dietary calcium and phosphorus, on calcium, phosphorus, nitrogen and fat metabolism in children. Amer. J. clin. Nutrition, 14, 76-82.

Matril K. F., I946. The digestibility of fats. A correlation of experimental data. Oil, 28, 344 .

MatTson F. H., Lutron E. S., I958. The specific distribution of fatty acids in the glycerides of animal and vegetable fats. J. biol. Chem., 288, 868-871.

PetTerson 1964. Inverkan av hög kalciumgiva $\mathrm{p} 1$ totalfettets och de enskilda fettsyrornas smältbarhet hos växande svin. Lantbrukshögskolans meddelanden Serie $A, \mathrm{Nr}, \mathrm{I} 6,12 \mathrm{p}$.

Planche N. E., Crotre C., Mule A., Sarles H., r 966. Effets des variations des lipides alimentaires sur l'excrétion fécale des graisses chez le Rat. Rev. Franç. Études clin. biol., 11, 931-934.

Renner R., Hili F. W., 1958. Metabolizable energy values of fats and fatty acids for chickens. Proc. Cornell Nutr. Conf., 95-100.

RENNER R., HILL F. W., I96r. Factors affecting the absorbability of saturated fatty acids in the chick. J. Nutr., 74, 254-258.

Richards J. F., Carroll K. K., 1959. Studies on calcium phosphate fatty acid salts occuring in fecal lipids. Can. J. biochem. physiol., 37, 725-730.

SAMmons H. G., Wiggs S. M., I 960 . The separation estimation and analysis of calcium soaps in human faeces. Clin. chim. Acta., 5, I4I-145.

Savary P., r966. Sur la solubilisation micellaire des acides palmitique, stéarique et oléique dans les solutions d'acides biliaires conjugués. Biochim. biophys. Acta, 126, 328-334.

Savary P., Constantin M. J., I967. Sur la solubilisation micellaire de l'hexadécane et son passage dans la lymphe thoracique du Rat. Biochim. biophys. Acta, 187, 264-276.

Savary P., Constantin M. J., Desnuelle P., i $96 \mathrm{r}$. Sur la structure des triglycérides des chylomicrons lymphatiques du Rat. Biochim. biophys. Acta, 48, 562-571. 
Savary P., Desnuelle P., 1961. Sur la répartition des chaînes saturées et insaturées entre les positions externes et internes des triglycérides mixtes végétaux. Biochim. biophys. Acta, 50, 319-324.

Savary P., Flanzy J., Dosnuelle P., 1957. Emploi de la lipase pancréatique pour l'étude de la structure des curps gras naturels. Biochim. biophys. Acta, 24, 414-423.

Scribante P., Favarger P., I954. Etude de la digestibilité de l'acide stéarique et de ses esters glycéridiques chez le Rat. Helv. physiol. pharmac. Acta, 12, 74-89.

Swell L., Trout Jr. E. C., Freld Jr. H., Treadwell C. R., 1956. Occurence and nature of a fecal phosphorus-containing lipid. J. biol. Chem., 228, 743-75०.

Thinulis C., 1968. Les divers facteurs influant sur l'utilisation digéstive des matières grasses. Ann. Nutr Alim. 28, 245-258).

Toullec R., Flanzy J., Rigaud J., I968. Note sur le dosage des lipides des fèces. Ann. Biol. anim. Bioch. Biophys. (sous presse).

Vodovar N., Flanzy Y., rg66. Mécanisme de la pénétration et voie d'acheminement des graisses dans la cellule absorbante de l'intestin grêle du Porc. Ann. Biol. anim. Bioch. Biophys., 6, 31 5-332.

WERner M., 1965. Vergleichende Bilanzuntersuchungen der Fettverdauung mit chemischen Methoden und radioaktiven Lipiden. Helv. med. Acta, 82, 457-460.

Werner M., Lutwak L., 1963. Dietary (fat and calcium) influences on fat absorption, and their kinetic interpretation. Fedn. Proc. 22, 553.

Williams J. N., I962. A Simple method for extraction of intact lipids from fæces. J. Lab. clin. Med. 60, $839-845$.

Yacowitz H., Fleischman A. I., Amsden R. T., Bierenbaum M. L., Ig67. Effects of dietary calcium upon lipid metabolism in rats fed saturated or insaturated fat. J. Nutr., 82, 389-392.

Young R. J., GarReTT R. L., I963. The effect of environment, diet composition and the ratio of fatty acids in the mixture on the absorption of fatty acids by the chick. Proc. Cornell Nutr. Conf., 7I-79. 